\section{SP0017 LESSONS LEARNED FROM LYME DISEASE: ONLY PERSISTENT BACTERIA OR AUTOIMMUNITY}

B Huber. Pathology, Tufts University School of Medicine, Boston, USA

10.1136/annrheumdis-2001.81

Antibiotic treatment-resistant Lyme arthritis is a chronic inflammatory joint disease, associated with a limited number of MHC alleles, including HLA DRb1*0401. A marked antibody and T cell response to outer surface protein A (OspA) of Borrelia burgdorferi, the causative agent of Lyme disease, is often seen during prolonged episodes of arthritis. An autoimmune mechanism has been implicated due to the lack of the Borrelia burgdorferi antigen in the target organ and apparent cross-reaction at the $\mathrm{T}$ cell level between the bacterial OspA and a self-protein, LFA-1aL. To directly test this hypothesis, we isolated and cloned T cells specific for the immunodominant epitope of OspA, OspA165-174, from five DRb1*0401+ patients, using OspA-class II tetramers. These clones were tested for their ability to respond to peptide containing the human homologue of OspA165-174, LFA$1 \mathrm{aL} 332-340$, as determined by proliferation and cytokine production. All of the $\mathrm{T}$ cell lines responded to minute concentrations of OspA165-174 by proliferation and secretion o IFN-g and IL-13. In contrast, larger concentrations of LFA-1aL326345 were required for stimulation, and only $10 \%$ of the clones proliferated vigorously. With this stimulation, approximately half of the cell lines secreted IL-13 but produced no IFN-g. LFA-1aLtetramers required a 2.5 - to 25 -fold higher concentration to stain dual reactive clones than OspA tetramer. Our analysis at the clonal level demonstrate that LFA-1aL326-345 behaves as an analogue for the OspA peptide, but a greater concentration of the LFA-1 peptide is required and an altered cytokine response is elicited. Thus, a single bacterial-specific $\mathrm{T}$ cell may respond to a cross-reactive human protein, LFA-1aL, indicating a role for molecular mimicry, and thus, autoimmunity, in treatment resistant Lyme arthritis.

\section{Review session $\mathrm{N}^{\circ} 2$ - Thursday 14 June, 10.0011.45/South Hall}

\section{SP0019 HOW THE CLINICIAN CAN USE THE COCHRANE LIBRARY}

${ }^{1} \mathrm{P}$ Tugwell, ${ }^{2} \mathrm{~J}$ McGowan, ${ }^{3} \mathrm{~V}$ Welch, ${ }^{4} \mathrm{~B}$ Shea, ${ }^{5} \mathrm{G}$ Wells. ${ }^{1}$ Department of Medicine; ${ }^{2}$ Library Services, The Ottawa Hospital; ${ }^{3}$ Clinical Epidemiology Unit, Loeb Research Institute, Ottawa, Canada; ${ }^{4}$ Institute of Population Health, University of Ottawa; ${ }^{5}$ Department of Epidemiology and Community Medicine

10.1136/annrheumdis-2001.82

Objectives To demonstrate online searching of The Cochrane Library for clinicians and illustrate how The Cochrane Library can be used in practice.

The Cochrane Library is the single best source of updated systematic reviews of health care interventions. There are now 37 published Cochrane reviews relating to Musculoskeletal Diseases. The abstracts can be accessed for free on the internet and lay summaries written in consultation with consumers are also available on the internet (www.arthritis.ca). The first part of this workshop will demonstrate how to use text words to search for consumer and scientific abstracts available for free on the internet. In addition, the web interface for the full Cochrane Library will be shown.
The second purpose of this workshop is to provide examples of how to use Cochrane reviews in clinical practice. For example, the University of Ottawa has developed hospital-wide access to the Cochrane Library, and these can be accessed by clinicians from any computer with an internet connexion. The pooled estimate of efficacy and side effects is the best estimate from all published randomised controlled trials. The direction and statistical significance of this estimate can be easily interpreted. The second part of the workshop will illustrate the interpretation of the magnitude and direction of the pooled estimate. In addition, the workshop will illustrate how these estimates can be used as part of evidence based practice.

\section{SP0022 LOW BACK PAIN AND IMAGING}

N Boos. Chief of Spinal Surgery, Orthopaedic University Hospital Zurich, Zurich, Switzerland

10.1136/annrheumdis-2001.83

Low-back pain is a very common but benign and in general selflimiting disease indicating that only a small proportion of the patients will require sophisticated imaging studies. Recent studies have highlighted that a simple relation of structural abnormalities to low-back pain is impossible because similar alterations can be found in symptomatic as well as in asymptomatic individuals. These findings question our current criteria for the diagnosis of low-back pain disorders with regard to their discriminative power in differentiating diseased and non-diseased individuals. Structural abnormalities demonstrated by imaging studies should therefore only be interpreted in the light of the clinical findings. This review has shown that only a few studies contribute to our understanding of the clinical efficacy of imaging studies in the evaluation of low-back pain disorders. There is an absolute need for comprehensive well conducted studies on the impact of specific image modalities on diagnosis and treatment of lumbar spinal disease.

\section{Stem cell treatment of autoimmune disease - Thursday 14 June, 10.00-11.45/Meeting Hall I}

\section{SP0023 HAEMOPOIETIC STEM CELL TRANSPLANATION FOR SEVERE AUTOIMMUNE DISEASE}

A Tyndall. Rheumatology, University of Basel, Basel, Switzerland

10.1136/annrheumdis-2001.84

Reporting on behalf of the EULAR and EBMT Autoimmune Disease Stem Cell Project. High dose immunoablative therapy with autologous haemopoietic stem cell transplantation (HSCT) has been used to treat selected patients with severe autoimmune disease (AD) in over 400 cases world wide. 334 transplants (327 autologous) are registered in the combined EULAR/EBMT data base, target ADs and numbers being: multiple sclerosis-102, SSc70, RA-41, JIA-35, SLE-25, dermatomyositis-6, Bechets-3, vasculitis 6 and others. Patients were from 78 transplant centres in 22 countries with a median follow up of 16(1-81) months. Four basic conditioning regimens were used in phase I and II pilot studies, with an unmanipulated graft product in 85 cases. A 